\title{
Diagnóstico de comportamientos de riesgo en adolescentes y jóvenes universitarios
}

\section{Cecilia del Carmen Rojas Montoya - Irene Margarita Espinosa Parra - Miguel Ángel Herrera Herrera - Elvia Lizette Parra Jiménez - Diana Patricia Aguirre Ojeda - Yanko Sixtos Baldenebro - Mayra Consuelo Pérez Pimienta}

RESUMEN: El perfil epidemiológico en adolescentes y jóvenes es alarmante por el carácter prevenible de la morbimortalidad y sus repercusiones. El Consejo Nacional de Población (2010) menciona la importancia de elaborar un diagnóstico de la situación actual y diseñar enfoques de intervención. Se realizó una investigación cuantitativa, descriptiva, con muestra no probabilística por conveniencia, aplicándose el cuestionario YRBSS; se observó que las dimensiones de mayor riesgo fueron sedentarismo, alimentación, consumo de alcohol, conductas sexuales y accidentes de tráfico. Existió una correlación moderada entre el riesgo suicida y la violencia de pareja ( $\mathrm{r}=.583, \mathrm{p}=.002$ ), los riesgos a accidentes de tráfico con el consumo de tabaco $(\mathrm{r}=.435, \mathrm{p}=.016)$ y con el sedentarismo ( $\mathrm{r}=.406, \mathrm{p}=.026)$. Los resultados corroboran la necesidad de identificar las principales conductas de riesgo mediante un 
diagnóstico integral para brindar las herramientas necesarias y de esa manera disminuir conductas de riesgo que afecten el desarrollo personal, profesional así como su entorno familiar y social.

Palabras clave: conductas; riesgo; adolescentes; diagnóstico; violencia

ABSTRACT: The epidemiological profile in adolescents and young people is alarming because of the preventable nature of morbidity and mortality and their repercussions. The National Population Council (2010) mentions the importance of preparing a diagnosis of the current situation and designing intervention approaches. A quantitative, descriptive investigation was carried out, with a non-probabilistic sample for convenience, applying the YRBSS questionnaire; it was observed that the dimensions of greatest risk were sedentary lifestyle, food, alcohol consumption, sexual behaviors and traffic accidents. There was a moderate correlation between suicide risk and intimate partner violence $(r=.583, \mathrm{p}=.002)$, risks to traffic accidents with tobacco consumption $(r=.435, \mathrm{p}=.016)$ and sedentary lifestyle $(r=.406, \mathrm{p}=.026)$. The results corroborate the need to identify the main risk behaviors through a comprehensive diagnosis to provide the necessary tools and thus reduce risk behaviors that affect personal, professional development as well as their family and social environment.

Keywords: behavior; risk; adolescents; diagnosis; violence

\section{Introducción}

La Organización Mundial de la Salud en el 2016, refirió que la primera causa de mortalidad en adolescentes y jóvenes son los accidentes viales, principalmente aquellos en los que intervienen consumo de sustancias como el alcohol o drogas ilícitas; como segunda causa aquellas por complicaciones durante el embarazo y posterior él y como tercera el suicidio.

De acuerdo al Consejo Nacional de Población (CONAPO, 2010), las tendencias del perfil epidemiológico en este grupo de edad son alarmantes por el carácter prevenible de las causas de mortalidad, esto como consecuencia de los inadecuados estilos de vida.

Dentro de la causas de morbimortalidad se deben considerar factores y conductas que amenacen el bienestar y salud de los individuos. Celís y Vargas (2005) definen una conducta riesgosa como aquella manifiesta o intra-verbal que atente contra el equilibrio o el desarrollo biológico, psicológico y social de la persona (como se citó en Salas, 2018).

La CONAPO (2010) menciona la importancia de elaborar un diagnóstico que describa la situación de los adolescentes y jóvenes en el país; de esta manera se podrá diseñar el enfoque de intervención considerando los diversos contextos socioculturales y emocionales, así como los 
procesos propios de estas etapas del desarrollo. Dicho diagnóstico debe considerar las diferentes etapas de la adolescencia y la exposición a las conductas de riesgo; es decir, (Orbegoso , s.f., pág. 6) durante el periodo inicial (10 a 14 años) se comienza principalmente con el consumo de sustancias (alcohol y tabaco) así como los riesgos sexuales; en la etapa media (14 a 17 años) se genera un mayor involucramiento con las conductas de riesgo, aumentando también la asociación entre estas y por último en la etapa tardía (hasta los 19 años) con base en las experiencias previas estas conductas se intensificarán o disminuirán.

\section{Conductas de riesgo}

Jessor en 1991 (como se citó en Krauskopf, 2003) propone dos tipos de conducta de riesgo, siendo la primera aquellas que comprometen el aspecto del desarrollo psicosocial o la supervivencia de la persona, buscando el peligro en sí; el segundo tipo son las que involucran riesgos, propias del grupo de edad, las cuales se asumen de manera consciente y como parte del compromiso y la necesidad de enriquecimiento de experiencias formadoras y vivenciales.

En las primeras conductas de riesgo destaca la sobreestimación de la invulnerabilidad, haciendo creer a los individuos que no habrá consecuencias por llevar a cabo la acción. Siendo estas parte de las principales causas de mortalidad e incapacidad en el grupo adolescente y juvenil. Se calcula que en 2015 murieron 1,2 millones de adolescentes, es decir, más de 3000 al día, en su mayoría por causas prevenibles o tratables (Organización Mundial de la Salud [OMS], 2018).

Cifras de la OMS refieren que a nivel mundial la primera causa de mortalidad siguen siendo los accidentes viales, principalmente aquellos en que los adolescentes y jóvenes se encuentran bajo la influencia de sustancias como el alcohol; la segunda causa son las complicaciones durante el embarazo y el parto entre los 15 y 19 años; y como tercera el suicidio. Asimismo, más de 2 millones de adolescentes en el mundo viven con VIH (World Health Organization, 2016).

En México, el Instituto Nacional de Geografía y Estadística (INEGI, 2016) reportó que las principales causas de mortalidad de 15 a 24 años son, en primer lugar, los accidentes de tráfico; en segundo, las agresiones y, en tercero, las lesiones autoinfligidas. En Nayarit, las cifras son semejantes, sin embargo, en tercer lugar están los tumores malignos y en cuarto las lesiones autoinfligidas. Dentro de las principales cifras relacionadas a salud se encuentra el consumo de tabaco y alcohol, donde 9 de cada 100 adolescentes refirieron haber fumado 100 cigarrillos en su vida, lo que representa el 12.3\% de prevalencia con una edad promedio de inicio de 14.6 años. Respecto al alcohol, donde 25 de cada 100 han tomado bebidas alcohólicas en el último año con una prevalencia de 25\%. De estos, 11 de cada 100 han consumido más de 5 copas en el último mes (Gutiérrez et al., 2012). 
El tema de salud reproductiva se enfoca principalmente a conductas de riesgo, teniendo un aumento de prevalencia de $23 \%$ con el inicio de vida sexual activa entre los 10 y los 19 años, de los cuales existió una disminución de porcentaje respecto al uso de protección en el inicio de vida sexual activa, de 79\% a 33.4\% en mujeres y de $47.5 \%$ a $14.4 \%$ en hombres. La atención por enfermedades de transmisión sexual se encontró en 2.3\%. Lamentablemente, la tasa de fecundidad aumentó a 37.0 nacimientos por cada 1000 mujeres en edad adolescente, y, del total de aquellas que iniciaron su vida sexual activa entre los 10 y 19 años, el 51.9\% refiere haber estado embarazada y el 10.7\% lo estaba al momento de la encuesta (Gutiérrez et al., 2012).

El consumo de alcohol o tabaco, la falta de actividad física, las relaciones sexuales sin protección y/o la exposición a la violencia pueden poner en peligro no solo su salud actual, sino también la de su adultez e incluso la salud de sus futuros hijos (OMS, 2018).

En 2010, uno de cada 5 jóvenes accedía a la educación superior en México, lo que exige a las universidades constituirse en un espacio ideal para implementar medidas que redunden en la mejora y mantención de las condiciones de salud de los jóvenes (Hidalgo-Rasmussen, 2014).

Como Instituciones formadoras se asume la responsabilidad de educar con base en un modelo integral, otorgando las bases no solo académicas sino formadoras de estilos de vida saludable (Universidad Autónoma de Nayarit [UAN], 2016).

Es así que conociendo qué conductas de riesgo presentan los adolescentes y jóvenes universitarios y basándonos en las teorías de riesgo, protección y resiliencia se puede determinar qué estrategias de intervención implementar. La intervención en la teoría de riesgo, como lo menciona Salas (2018), se enfoca en trabajar con los factores y conductas de riesgo, previniendo las consecuencias; en cambio las enfocadas en la protección y resiliencia potencia los factores y conductas protectoras del individuo y comunidad, aumenta de esta manera la eficacia preventiva. El objetivo principal de la investigación es determinar los principales comportamientos de riesgo en adolescentes y jóvenes de la Universidad Autónoma de Nayarit.

\section{Metodología}

Se realizó una investigación de tipo cuantitativa, descriptiva, transversal. Se seleccionó una muestra no probabilística por conveniencia, teniendo como criterio de selección alumnos de nuevo ingreso al ciclo escolar 2016-2017, con edad de 17 a 25 años, de las licenciaturas en Psicología y Educación Infantil de la Universidad Autónoma de Nayarit. Se consideró criterio de eliminación no ser alumno inscrito a las licenciaturas mencionadas y como criterio de exclusión no haber realizado en su totalidad el instrumento. 
Mediante previo consentimiento informado y respetando los aspectos éticos de confidencialidad se aplicó el instrumento "Youth Risk Behaviours Survey (YRBSS)" que consta de 100 ítems de opción múltiple, el cual se enfoca en 7 conductas de riesgo como son accidentes de tránsito, violencia, comportamientos sexuales de riesgo, trastornos de alimentación, actividad física/sedentarismo, suicidio, consumo de sustancias legales e ilegales (Centers for Disease Control and Prevention, 2013).

El instrumento, elaborado en 1989 por el Centers for Disease Control and Prevention (CDC), ha reportado evidencia de su confiabilidad y validez (Brener, Billy y Grady, 2003; Brener, Kann, McManus, Kinchen, Sundberg y Ross, 2002). Dicho instrumento se aplicó en formato digital con conexión a plataforma, mediante el proceso previamente descrito. Se realizó un análisis estadístico por medio del programa SPSS versión 20.0 obteniéndose estadísticos descriptivos, de correlación y discriminantes.

\section{Resultados}

Se aplicó el instrumento YRBSS a un total de 139 alumnos, predominando con un $78.4 \%$ las mujeres. Debido a la matrícula de los programas académicos, el 77\% pertenecía a la licenciatura de Psicología. El grupo de edad predominante fue el de 17 a 19 años con un 84.17\%.

\section{Dimensiones de conductas de riesgo}

Se recodificaron las respuestas de las y los participantes categorizándolas en puntajes para su análisis: 1) conducta sin riesgo, 2) conducta de poco riesgo, 3) conducta de medio riesgo, y 4) conducta de alto riesgo. A partir de esta homogeneización, se agruparon las variables por categorías de riesgo y se realizaron pruebas de diferencia y de correlación para una mayor comprensión de los resultados. Los puntajes obtenidos por categoría fueron los siguientes:

Tabla 1

Puntajes* de dimensiones de conductas de riesgo

\begin{tabular}{|l|c|c|}
\hline Dimensiones de conductas de riesgo & Media & Desviación típica \\
\hline Riesgos a accidentes de tráfico & 1.675 & 433 \\
\hline Violencia & 1.036 & .107 \\
\hline Violencia de pareja & 1.151 & .491 \\
\hline Riesgo suicida & 1.358 & .603 \\
\hline Acoso escolar & 1.151 & .488 \\
\hline Consumo de tabaco & 1.520 & .749 \\
\hline Consumo de alcohol & 1.864 & .709 \\
\hline Consumo de marihuana & 1.172 & .542 \\
\hline
\end{tabular}




\begin{tabular}{|l|c|c|}
\hline Dimensiones de conductas de riesgo & Media & Desviación típica \\
\hline Consumo de otras drogas & 1.034 & .159 \\
\hline Conductas sexuales de riesgo & 1.727 & .411 \\
\hline Riesgos de la alimentación & 2.262 & .421 \\
\hline Sedentarismo / Actividad física & 2.301 & .550 \\
\hline
\end{tabular}

Nota: Rango mínimo y máximo 1 a 4.

De lo anterior, se observa que las dimensiones de mayor riesgo reportadas fueron a) sedentarismo, b) riesgos de la alimentación, c) consumo de alcohol, d) conductas sexuales de riesgo y e) riesgos a accidentes de tráfico. Segmentado por sexo, el análisis de las categorías es el siguiente

Tabla 2

Diferencias significativas por sexo en puntajes* de riesgo

\begin{tabular}{|l|c|c|c|}
\hline \multicolumn{1}{|c|}{ Dimensiones de conductas de riesgo } & Masculino & Femenino & $\begin{array}{c}\text { Z diferencia } \\
\text { significativa }\end{array}$ \\
\hline Riesgos a accidentes de tráfico & 1.680 & 1.674 & \\
\hline Violencia & 1.045 & 1.034 & \\
\hline Violencia de pareja & 1.083 & 1.17 & \\
\hline Riesgo suicida & 1.280 & 1.38 & \\
\hline Acoso escolar & 1.100 & 1.165 & \\
\hline Consumo de tabaco & 1.678 & 1.477 & \\
\hline Consumo de alcohol & 2.060 & 1.81 & \\
\hline Consumo de marihuana & 1.311 & 1.134 & $.--2,204^{* *}$ \\
\hline Consumo de otras drogas & 1.040 & 1.032 & \\
\hline Conductas sexuales de riesgo & 1.748 & 1.721 & \\
\hline Riesgos de la alimentación & 2.680 & 2.607 & \\
\hline Sedentarismo / Actividad física & 2.025 & 2.376 & $.-3,251^{* *}$ \\
\hline
\end{tabular}

Nota: **: p=>.028 *: Rango mínimo y máximo 1 a 4.

De esto se rescata que la construcción cultural del género tiene su efecto en las conductas de riesgo, donde las mujeres puntuaron de manera significativa a mayor riesgo en conductas relacionadas con el sedentarismo ( $\mathrm{z}=.-3,251, \mathrm{p}=.001)$, mientras que los hombres lo hicieron en los comportamientos referentes al consumo de marihuana ( $\mathrm{z}=.--2,204, \mathrm{p}=.028$ ).

Además del sexo, se encontraron diferencias significativas a partir de dos variables: la carrera estudiada y el grupo de edad. En materia de la licenciatura, las y los estudiantes en Educación Infantil reportaron mayor índices de riesgo en las conductas sexuales de riesgo ( $\mathrm{z}=.-1,506$, $\mathrm{p}=.001)$ y en sedentarismo $(\mathrm{z}=.-2,408, \mathrm{p}=.016)$, relacionado esto probablemente con el perfil de 
ingreso a esa área de conocimiento, mientras que desde el grupo de edad, se encontraron diferencias significativas en el consumo de tabaco $(\mathrm{z}=.-2,302, \mathrm{p}=.021)$ y marihuana $(\mathrm{z}=.-2,343$, $\mathrm{p}=.019)$ teniendo el grupo de edad de 20 a 25 años un mayor riesgo en el consumo de estas sustancias, justificando la relevancia de profundizar en las elecciones y situaciones de vida que ha llevado a estas personas a ingresar a una carrera universitaria en esta etapa de vida.

Respecto a la relación entre las dimensiones, se encontraron correlaciones positivas estadísticamente significativas en nivel de 0.00 bilateral, entre el consumo de tabaco con el consumo de alcohol ( $r=.585)$ y marihuana ( $r=.505)$, y esta última con el consumo de otras drogas $(r=.510)$, evidenciando un policonsumo en los y las estudiantes.

Segmentado por variables sociodemográficas, el análisis generó, en el caso de los hombres, correlaciones estadísticamente significativas positivas entre riesgo suicida y la violencia de pareja ( $r=.583, p=.002$ ), el consumo de tabaco con los riesgos a accidentes de tráfico $(r=.435$, $\mathrm{p}=.016)$ y el consumo de tabaco con el sedentarismo $(\mathrm{r}=.406, \mathrm{p}=.026)$.

En el caso de la carrera en Educación Infantil, correlacionaron de manera positiva y estadísticamente significativa el acoso escolar y la violencia de pareja ( $r=.506, p=.003)$, la conducta sexual de riesgo y el consumo de drogas $(r=.412 \mathrm{p}=.019)$ y el sedentarismo con los riesgos a la alimentación ( $\mathrm{r}=.519, \mathrm{p}=.002)$.

En el grupo de 20 a 25 años, se encontraron correlaciones positivas estadísticamente significativas entre la violencia de pareja y el acoso escolar $(r=.673, \mathrm{p}=.000)$ y consumo de drogas $(r=.419, p=.047)$ y entre los riesgos a la alimentación y el consumo de alcohol $(r=.505, p=.014)$ y entre los riesgos de accidentes de tráfico con el consumo de alcohol ( $r=.564$, $p=.005)$.

\section{Conclusiones}

Realizando un análisis se puede observar que el consumo de sustancias legales e ilegales sigue siendo una de las principales conductas de riesgo; con base en la Encuesta Nacional de Consumo de Drogas, Alcohol y Tabaco (2016) resalta el bajo porcentaje de percepción de riesgo al consumir sustancias como la cocaína (62.8\%), inhalables (66.9\%) y marihuana (18.7\%). Destaca la presencia de trastornos en la salud mental como depresión, ansiedad, así como ideas suicidas; esto corroborado por la prevalencia (INEGI, 2016) siendo la tercera causa de mortalidad en el estado de Nayarit.

Los resultados presentados corroboran la necesidad de identificar las principales conductas de riesgo con base en factores biológicos, ambientales, de comportamiento, y socioculturales. Al 
tener un diagnóstico integral, de los estudiantes de la Universidad Autónoma de Nayarit, pretendemos brindar las herramientas necesarias para incrementar factores de protección y resiliencia y así disminuir factores de riesgo que eviten conductas de riesgo que afecten el desarrollo personal, profesional así como su entorno familiar y social.

\section{Referencias}

Brener, N., Billy, J., y Grady, W. (2003). Assessment of factors affecting the validity of selfreported health-risk behavior among adolescents:evidence from the scientific literature. Journal of Adolescent Health, 33(6), 436-457.

Brener, N., Kann, L., McManus, T., Kinchen, S., Sundberg, E., y Ross, J. (2002). Reliability of the 1999 Youth Risk Behavior Survey. Journal of Adolescent Health, 31(4), 336-342.

Centers for Disease Control and Prevention. (2011). Youth Risk Behavior Surveillance-United. Centers for Disease Control and Prevention. Recuperado el Diciembre de 2015, de http://www.cdc.gov/mmwr/pdf/ss/ss6104.pdf

Centers for Disease Control and Prevention. (2013). Methodology of the Youth Risk Behavior Surveillance System. Centers for Disease Control and Prevention. Recuperado de: http://www.cdc.gov/mmwr/pdf/rr/rr6201.pdf

Consejo Nacional de Población. (2010). Situación actual de las y los jóvenes en México: diagnóstico sociodemográfico. Distrito Federal: CONAPO. Recuperado de https://bit.ly/2tHkTaS

Instituto Nacional de Psiquiatría Ramón de la Fuente Muñiz, Instituto Nacional de Salud Pública, Comisión Nacional contra las adicciones y Secretaría de salud (2017). Encuesta Nacional de Consumo de drogas, alcohol y tabaco 2016-2017. Ciudad de México:INPRFM. Recuperado de https://bit.ly/2CBfWk5

Gutiérrez, J., Rivera-Dommarco, J., Franco, A., Shamah Levy, T., Cuevas Nasu, L., Villalpando, S., y Romero , M. (2012). Encuesta Nacional de Salud y Nutrición 2012. Resultados Nacionales. Cuernavaca, México: Instituto Nacional de Salud Pública .

Instituto Nacional de Geografía y Estadística . (2016). Principales causas de mortalidad por residencia habitual, grupos de edad y sexo del fallecido. Recuperado de https://bit.ly/1syUvGY 
Jackson, C., Henderson, M., Frank, J., \& Haw, S. (2012). An overview of prevention of multiple risk behaviour in adolescence and young adulthood. Journal of public Health, 34(S1), 31-40. Krauskopf, D. (2003). Juventud,riesgo y violencia. Dimensiones de la violencia. Seminario permanente sobre violencia programa de las Naciones Unidas para el Desarrollo. El Salvador: Ed. PNUD.

Observatorio de los riesgos y calidad de vida. (2014). Observatorio de los riesgos y calidad de vida. Universidad de Guadalajara campus sur. Recuperado de http://www.cusur.udg.mx/circav/Que_es_y_como_funciona.html

Orbegoso , B. I. (s.f.). Conductas de riesgo convencionales. Obtenido de Seguridad Ciudadana de las redes sociales. Recuperado de http://www.cedro.org.pe/cursoonline20134/descargas/Modulo_2.pdf

Organización Mundial de la Salud . (5 de Febrero de 2018). Adolescentes: riesgos para la salud y soluciones. Recuperado de https://bit.ly/2KLpYD1

Organización Mundial de la Salud. (2000). La salud de los jóvenes: un desafío para la sociedad. Ginebra, Suiza: OMS. Recuperado de https://bit.ly/2YeuAJh

Salas, F. G. (2018). Caracterización de factores implicados en las conductas de riesgo en adolescentes. $A B R A, 38(56), 1-16$.

Universidad Autónoma de Nayarit. (2016). Plan de Desarrollo Institucional 2016-2022 de la Universidad Autónoma de Nayarit. Gaceta Universitaria. Recuperado de: http://www.uan.edu.mx/es/avisos/plan-de-desarrollo-institucional-2016-2022

World Health Organization. (Mayo de 2016). Adolescents:health risks and solutions. Recuperado de World Health Organization: http://www.who.int/mediacentre/factsheets/fs345/en/ 